\title{
Influence of reversal with sugammadex or neostigmine on post-operative quality of recovery following a single bolus dose of rocuronium: a prospective, randomised, double-blinded, controlled study
}

Sun-Joon Bai ${ }^{1}$, Ki-Young Lee ${ }^{1}$, Sung Soo Kim ${ }^{2}$, Jung Hwa Hong ${ }^{3}$, Hoon Jae Nam ${ }^{1}$, Jae Chul Koh ${ }^{4, *}$, Na Young $\mathrm{Kim}^{1, *}$

${ }^{1}$ Department of Anaesthesiology and Pain Medicine, Anaesthesia and Pain Research Institute, Yonsei University College of Medicine, 50-1 Yonsei-ro, Seodaemun-gu, Seoul 03722, Republic of Korea; sjbae@yuhs.ac (S.J.B.); kylee504@yuhs.ac (K.Y.L.); hjnam90@yuhs.ac (H.J.N.); knnyyy@yuhs.ac (N.Y.K.)

${ }^{2}$ Institute of Vision Research, Department of Ophthalmology, Yonsei University College of Medicine, 50-1 Yonsei-ro, Seodaemun-gu, Seoul 03722, Republic of Korea; semekim@yuhs.ac

${ }^{3}$ Department of Policy Research Affairs National Health Insurance Service Ilsan Hospital, Goyang, Gyeonggi-do, Republic of Korea; jh_hong@nhimc.or.kr

${ }^{4}$ Department of Anaesthesiology and Pain Medicine, Korea University Anam Hospital, Korea University College of Medicine, 73, Inchon-ro, Seongbuk-gu, Seoul 02841, Republic of Korea.; jaykoh@korea.ac.kr

\section{Corresponding author:}

*Jae Chul Koh, MD, PhD

Department of Anaesthesiology and Pain Medicine, Korea University Anam Hospital, Korea University College of Medicine, 73, Inchon-ro, Seongbuk-gu, Seoul 02841, Republic of Korea. Tel: (+82) 2920 5632; Fax: (+82) 22227 6517; E-mail: jaykoh@korea.ac.kr

*Na Young Kim, MD, PhD

Department of Anaesthesiology and Pain Medicine, Anaesthesia and Pain Research Institute, Yonsei University College of Medicine, 50-1 Yonsei-ro, Seodaemun-gu, Seoul 03722, Republic of Korea.

Tel: (+82) 22228 4435; Fax: (+82) 22227 7897; E-mail: knnyyy@yuhs.ac 


\begin{abstract}
There is a lack of data comparing sugammadex with anticholinesterase for the quality of anaesthesia recovery, especially following a single bolus dose of rocuronium. Thus, we evaluated the influence of reversal with sugammadex or neostigmine on post-operative quality of recovery by using the Post-operative Quality Recovery Scale (PQRS). A total of 86 patients undergoing trans-pars plana vitrectomy (TPPV) under general anaesthesia were intubated following a single bolus dose of rocuronium $(0.6 \mathrm{mg} / \mathrm{kg})$. At the end of surgery, patients were received either neostigmine or sugammadex. The quality of recovery was assessed using the PQRS at 15 minutes and 40 minutes after surgery, and on post-operative day 1 . The recovery rate in the physiological domain was higher in the sugammadex group at 15 minutes after surgery $(P=0.02)$. Though there were no significant differences in the overall cognitive recovery domain, patients in the sugammadex group could recall more numbers in reverse order. However, there were no significant differences between the groups in the other domains of the PQRS. The use of sugammadex may increase the quality of the post-operative physiological recovery at early post-operative periods compared with neostigmine use following a single bolus dose of rocuronium in patients undergoing TPPV with general anaesthesia.
\end{abstract}

Key words: anaesthesia, general; anaesthesia recovery period; single dose of rocuronium; sugammadex; neostigmine

Trial Registration: Registered at ClinicalTrials.gov; ttps:/clinicaltrials.gov/ct2/show/NCT03108989; Registration number NCT03108989 


\section{Introduction}

The quality of recovery is a crucial factor that must be evaluated in patients subjected to surgery under general anaesthesia. [1] Unlike the past, which focused exclusively on evaluating the outcome of surgery, postoperative quality of recovery has recently become an important issue. [1] Protracted and poor recovery after surgery can lead to delayed hospital discharge, increased costs, and delayed resumption of daily activity. [2] Particularly, in recent years, surgical procedures are increasingly being performed under outpatient settings rather than during hospitalization. [3] Therefore, anaesthetic goals have evolved focusing on early and explicit postoperative recovery.

Sugammadex, a $\gamma$-cyclodextrin derivative, has been approved as the first targeted agent to remove steroid neuromuscular (NM) blocking agents by forming a chemical compound. [4] This agent has contributed to anaesthesia protocols by enabling effective and complete reversal of NM blockade. [5,6] Besides the fast reversibility of NM blockade, advantageous effects of this agent on recovery components after anaesthesia such as consciousness, nausea/vomiting, or physiologic factors have been reported. [7-9] Nonetheless, controversial results on the superiority of sugammadex over conventional NM blockade reversal agents have been reported. $[10,11]$

The Post-operative Quality Recovery Scale (PQRS) assesses the quality of postoperative recovery over time using six domains of recovery. [12] This scale has been translated into different languages and has been used successfully to assess the quality of recovery after surgery. $[13,14]$ So far, there is a lack of data comparing sugammadex with anticholinesterase for the quality of anaesthesia recovery, especially following a single bolus dose of rocuronium.

Therefore, we evaluated the influence of reversal with sugammadex or neostigmine on 
post-operative quality of recovery following a single bolus dose of rocuronium by using the PQRS in patients undergoing TPPV. 


\section{Materials and Methods}

\section{Study design}

The study was designed as a prospective, double blind, randomised controlled trial. This study was approved by the Institutional Review Board and Hospital Research Ethics Committee of Severance Hospital (ref: 4-2015-0641) and registered at clinicalTrials.gov (ref: NCT03108989, April 11, 2017). Between February and July 2017, 90 patients aged over 60 years with an American Society of Anaesthesiologists (ASA) physical status of I or II who were scheduled to undergo TPPV under general anaesthesia, were enrolled in this study after providing written informed consent. The patients were free to withdraw from the study whenever they wished. Patients with the following conditions were excluded: known NM disease, significant renal or hepatic dysfunction, history of malignant hyperthermia, allergic reaction to sugammadex or rocuronium, on medication which can affect NM blockade such as anti-convulsants, magnesium, or a body mass index $>30 \mathrm{~kg} \mathrm{~m} \mathrm{~m}^{-2}$. Patients with psychological or language problems that might impede assessment of the PQRS were also excluded from this study.

\section{Randomisation and allocation}

The patients were randomized to either the neostigmine group (group $\mathrm{N}, \mathrm{n}=45$ ) or the sugammadex group (group $\mathrm{S}, \mathrm{n}=45$ ) using a computer-generated randomization table without performing blocks. A total of $3 \mathrm{mg}$ of study drug was prepared for the patients. For patients in group $\mathrm{N}$, a solution of neostigmine methylsulfate $1 \mathrm{mg}(0.5 \mathrm{mg} / \mathrm{mL}$ in a $1 \mathrm{~mL}$-vial; Daihan Pharm, Seoul, Korea) and glycopyrrolate $0.2 \mathrm{mg}(0.2 \mathrm{mg} / \mathrm{mL}$ in a $1 \mathrm{~mL}$-vial; Reyon Pharm, Seoul, Korea) was prepared, while a solution of sugammadex sodium $2 \mathrm{mg} / \mathrm{kg}$ (100 $\mathrm{mg} / \mathrm{mL}$ in a $2 \mathrm{~mL}$-vial; MSDKOREA, Seoul, Korea) and normal saline was prepared for patients in group S. The study drug was provided at the request of an anaesthesiologist who 
was blinded to the randomisation list. Both groups received study drugs intravenously at the end of surgery after confirmation of a train of four (TOF) ratio of 0.5 or greater. Three anaesthesiologists participated in the process, one, who was the aware of the patient assignment, prepared the drugs according to the assigned group. The others were investigators who were blinded to the group assignment and collected data and investigated patient outcomes only. The surgeons, recovery nurses, and patients were all blinded to the group assignment as well.

\section{Procedure}

Following the patient's arrival to the operating room, non-invasive blood pressure, electrocardiogram, pulse oxymetry, and bispectral index (Aspect A-2000 ${ }^{\circledR}$; Aspect Medical System Inc., Newton, MA) were monitored. Acceleromyography (TOF-Watch ${ }^{\circledR}$ SX; Organon Ltd, Dublin, Ireland) was monitored at the adductor pollicis muscle of the left arm for NM activity.

General anaesthesia was induced by an anaesthesiologist blinded to the group assignment. For loss of consciousness, propofol $1.5 \mathrm{mg} / \mathrm{kg}$ bolus and continuous infusion of remifentanil at $0.2 \mu \mathrm{g} / \mathrm{kg} / \mathrm{min}$ were administered. After confirmation of loss of consciousness, rocuronium $0.6 \mathrm{mg} / \mathrm{kg}$ was injected for NM blockade. Patients were manually ventilated using a laryngeal mask when spontaneous breathing disappeared. Endotracheal intubation was performed when the twitch response to TOF stimulation was absent. Mechanical ventilation was performed with $50 \%$ oxygen; tidal volume of $8 \mathrm{~mL} / \mathrm{kg}$ of predicted body weight, respiratory rate was adjusted to maintain end-tidal carbon dioxide between 30-35 $\mathrm{mmHg}$, and positive end-expiratory pressure of $5 \mathrm{mmHg}$. Anaesthesia was maintained with desflurane (0.7-1.0 age-adjusted minimal alveolar concentration) and continuous remifentanil infusion at $0.05-0.5 \mu \mathrm{g} / \mathrm{kg} / \mathrm{min}$ for target bispectral index values of hypnosis between $40-60$ 
and arterial pressure/heart rate within $20 \%$ of the baseline values. Temperature was maintained at $36-37^{\circ} \mathrm{C}$ with an air-warming blanket.

The surgeon performing the surgery was blinded to the group assignment. During the surgery, any agents for NM blockade were not administered. Twenty minutes before the end of surgery, propacetamol $2 \mathrm{~g}$ and ramosetron $0.3 \mathrm{mg}$ was administered for prevention of postoperative pain and nausea/vomiting (PONV). On termination of the procedure, both desflurane and remifentanil administration were stopped and TOF stimulation was performed. After confirmation of a train of four (TOF) ratio of 0.5 or greater, the NM blockade was reversed with the prepared study drug.

In the post-anaesthesia care unit (PACU), patients were monitored for oxygen saturation, heart rate, and blood pressure. The PQRS consisted of 6 domains of post-operative recovery (physiologic, nociceptive, emotive, cognitive, activities of daily living, and overall patient perspective). Each domain was assessed using the Korean version of the PQRS, which has been translated from previous publications of the PQRS. [12] For each patient, the following data were recorded: age, sex, height, weight, past medical history including hypertension, diabetes mellitus, cerebrovascular attack, pulmonary disease, and chronic kidney disease, and ASA physical status classification. Patients were assessed for baseline PQRS in 1-day before surgery, and then at 15 minutes, 40 minutes, and 1-day post-operatively. For each time point, the recovery was defined as "return to baseline values or better". The patient's response rate on overall patient perspective was assessed only at post-operative day 1 .

\section{Statistical analysis}

The primary endpoint of the current study was the evaluation of the effects of sugammadex compared with neostigmine on the recovery rate in physiological domain in patients who underwent TPPV with general anaesthesia. Based on a previous observational study, the 
sample size requirement was calculated to be at least 37 patients in each group with alpha of 0.05 and power of 0.9 to detect a 10.82 difference in odds ratio for the recovery rate of the physiologic domain between the two groups. [7] Considering a dropout rate of $20 \%, 45$ patients in each group were assessed for eligibility. Continuous demographic and study variables were expressed as mean (SD) or median (interquartile range) according to the Komogorov-Smirnov normality test. For categorical data, variables were expressed as proportion or number (percentage). Comparisons of continuous variables between groups were performed with the independent two-sample t-test and the Mann-Whitney U-test for parametric and non-parametric data, respectively. Categorical variables were compared using the chi-square test $\left(\chi^{2}\right)$ or the Fisher's exact test when appropriate. Data were analysed with SAS 9.4 (SAS Institute, Cary, NC, USA) and R software version 3.1.0. A $P$-value $<0.05$ was considered significant. 


\section{Results}

Overall, 92 patients were assessed for eligibility, 2 patients declined to participate and 90 patients were ultimately enrolled and randomly assigned into 2 groups; 84 patients completed the study. One and 3 patients in groups $\mathrm{N}$ and $\mathrm{S}$, respectively, dropped out of the study because of the unexpected change in anaesthesia method, respectively. In group S, one patient cancelled approval to participate after surgery and the other had a severe arrhythmic event and was dropped from the analysis. Therefore, 44 and 40 patients were ultimately analyzed in groups $\mathrm{N}$ and $\mathrm{S}$, respectively (Figure 1).

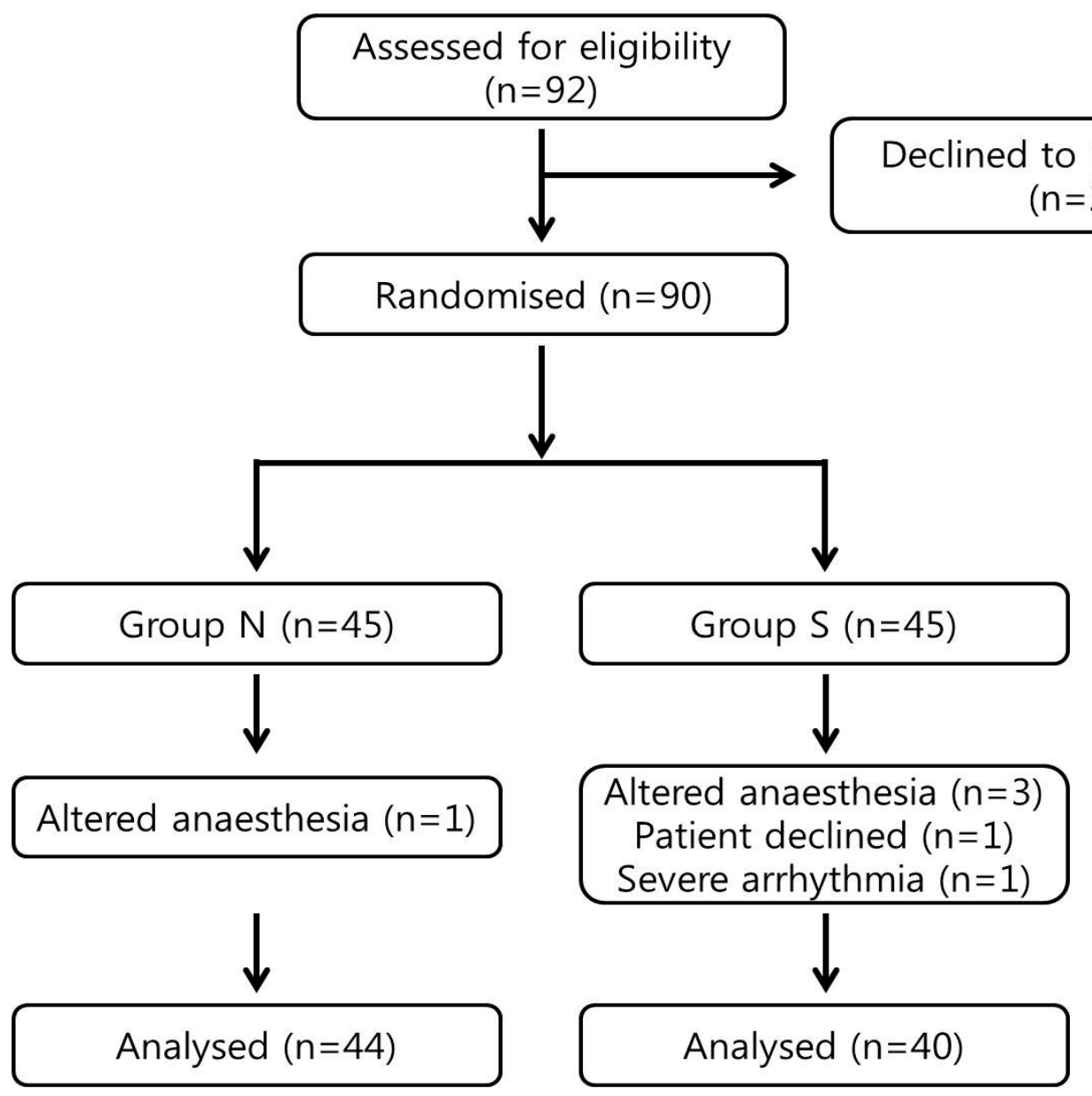

Figure 1. Flow chart indicating patient selection and exclusion criteria. group N: neostigmine was used for neuromuscular blockade reversal at the end of surgery; group S: sugammadex was used for neuromuscular blockade reversal at the end of surgery. 
Comparison of patient characteristics and perioperative drug use are presented in Table 1. There were more patients with ASA class II and the time from administration of study drug to extubation was significantly higher in group $\mathrm{N}$ than in group S. Differences in other variables were not found.

Table 1 Patients' characteristics and perioperative drug use.

\begin{tabular}{|c|c|c|c|}
\hline Characteristics & group $\mathrm{N}, n=44$ & group $\mathrm{S}, n=40$ & $\mathrm{P}$-value \\
\hline Age (year) & $63.5(60.5-66)$ & $64(61.5-67)$ & 0.581 \\
\hline Height $(\mathrm{cm})$ & $161.1(7.9)$ & $160.8(7.4)$ & 0.842 \\
\hline Weight $(\mathrm{kg})$ & $64.4(10.4)$ & $63.8(7.0)$ & 0.742 \\
\hline Sex female & $26(59 \%)$ & $26(65 \%)$ & 0.578 \\
\hline \multicolumn{4}{|l|}{ Past medical history } \\
\hline Hypertension & $23(52 \%)$ & $12(30 \%)$ & $0.039 *$ \\
\hline Diabetes mellitus & $10(23 \%)$ & $4(10 \%)$ & 0.118 \\
\hline Cerebrovascular attack & $3(7 \%)$ & $1(3 \%)$ & 0.618 \\
\hline Pulmonary disease & $1(2 \%)$ & $1(3 \%)$ & $>.999$ \\
\hline Chronic kidney disease & $4(9 \%)$ & $0(0)$ & - \\
\hline ASA class & & & $0.032 *$ \\
\hline I & $14(32 \%)$ & $22(55 \%)$ & \\
\hline II & $30(68 \%)$ & $18(45 \%)$ & \\
\hline \multicolumn{4}{|l|}{ Perioperative drug use } \\
\hline Rocuronium dose (mg) & $40(36-50)$ & $40(36.5-50)$ & 0.598 \\
\hline Total remifentanil used (sg) & $178(139-225)$ & $159(125-193.2)$ & 0.129 \\
\hline Time to extubation (sec) & $476.5(420-518.5)$ & $365(330-433.5)$ & $<0.001^{*}$ \\
\hline Total anaesthesia time (min) & $92.2(26.8)$ & $87.4(23.4)$ & 0.387 \\
\hline
\end{tabular}

Values are mean (SD), median (range) or number (\%). group N, neostigmine group; group S, sugammadex group; sec, second; min, minutes. ASA, American Society of Anaesthesiologists. $* \mathrm{P}<0.05$ between groups.

Table 2 shows the percentages of patients presenting overall recovery and recoveries by 
each domain of the PQRS at different time points. When compared with baseline values, there were no significant differences in the overall recovery of PQRS between the 2 groups at all time points post-operatively. As for the different domains of the PQRS, a significantly higher number of patients in group $\mathrm{S}$ showed recovery of the physiological domain 15 minutes after surgery than those in group $\mathrm{N}(95 \%$ vs $72 \%, P=0.02)$. No significant associations were found in the other PQRS domains between groups.

Table 2 Recovery rates of the Postoperative Quality Recovery Scale at different time points.

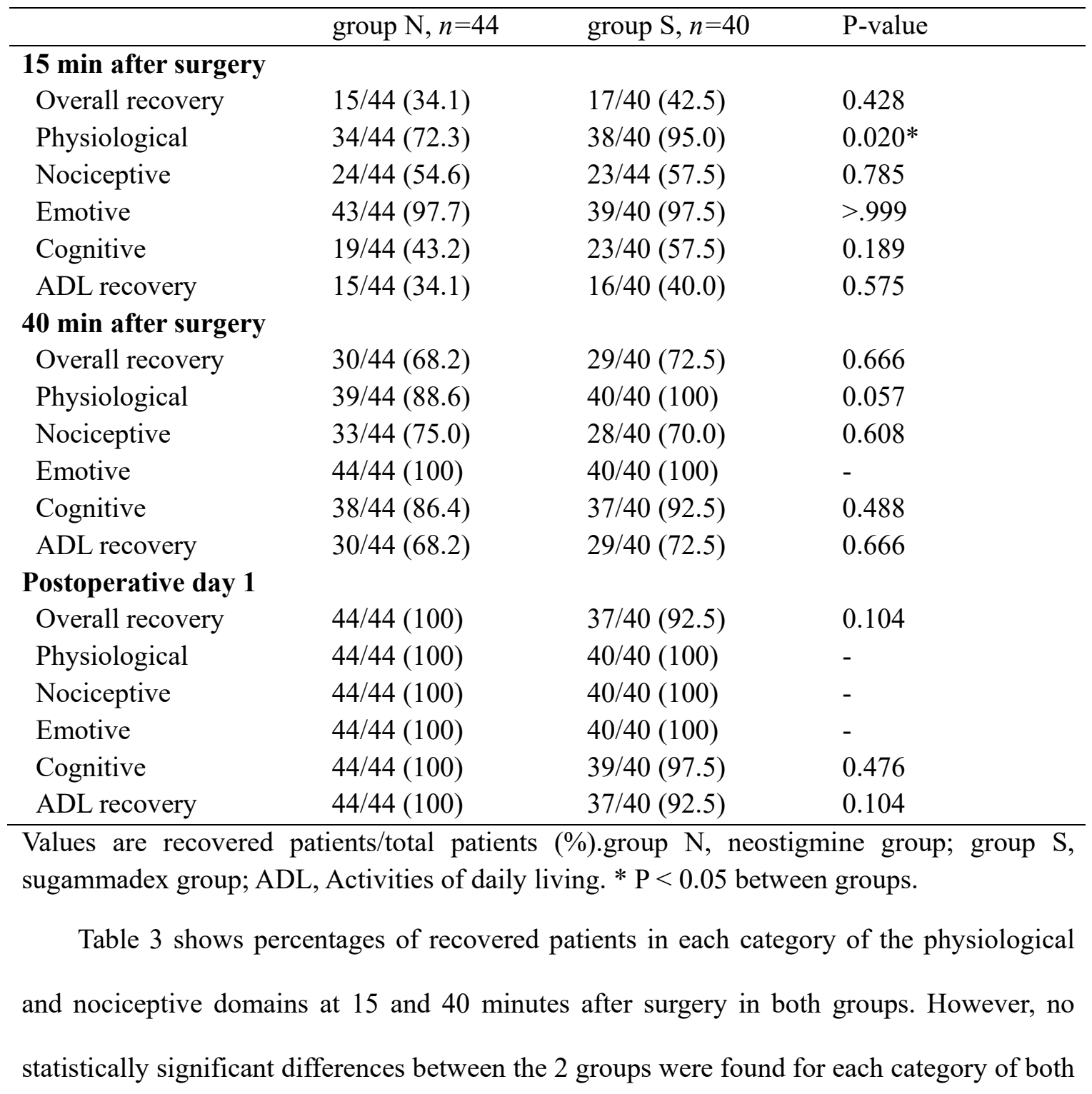


domains

Table 3 Percentages of recovered patients in each category of the physiological and nociceptive domains at 15 and 40 minutes after surgery.

\begin{tabular}{|c|c|c|c|}
\hline & group $\mathrm{N}, n=44$ & group $\mathrm{S}, n=40$ & P-value \\
\hline \multicolumn{4}{|l|}{15 minutes after surgery } \\
\hline \multicolumn{4}{|l|}{ Physiological } \\
\hline Blood nressure & 84.1 & 95 & 0.160 \\
\hline Dlood pressure & 93.2 & 100 & 0.243 \\
\hline Heart rate & 97.7 & 100 & $>0.999$ \\
\hline Temperature & 97.7 & 100 & $>0.999$ \\
\hline Respiration & 100 & 100 & - \\
\hline Oxygen use to maintain Spu2 & 100 & 100 & - \\
\hline $\begin{array}{l}\text { Aurway } \\
\text { Agitation }\end{array}$ & 97.7 & 100 & $>0.999$ \\
\hline $\begin{array}{l}\text { Agitation } \\
\text { Consciousness }\end{array}$ & 95.5 & 100 & 0.495 \\
\hline $\begin{array}{l}\text { Consciousness } \\
\text { Activity on command }\end{array}$ & 95.5 & 100 & 0.495 \\
\hline \multicolumn{4}{|l|}{ Nociceptive } \\
\hline Pain intensity & 56.8 & 60.0 & 0.768 \\
\hline Nausea and vomiting & 97.7 & 97.5 & $>0.999$ \\
\hline \multicolumn{4}{|l|}{40 minutes after surgery } \\
\hline \multicolumn{4}{|l|}{ Physiological } \\
\hline Blood pressure & 95.5 & 97.5 & $>0.999$ \\
\hline Deart rate & 95.5 & 100 & 0.495 \\
\hline Temperature & 100 & 100 & - \\
\hline Respiration & 100 & 100 & - \\
\hline Oxyoen use to maintain $\mathrm{SnO}_{2}$ & 100 & 100 & - \\
\hline Airway & 100 & 100 & - \\
\hline Agitation & 100 & 100 & - \\
\hline Consciousness & 97.7 & 100 & $>0.999$ \\
\hline Activity on command & 97.7 & 100 & $>0.999$ \\
\hline \multicolumn{4}{|l|}{ Nociceptive } \\
\hline & 75.0 & 70.0 & 0.633 \\
\hline Nausea and vomiting & 100 & 100 & - \\
\hline
\end{tabular}

No statistical differences were identified in the emotional and cognitive domain at 15 and 40 minutes after surgery between the 2 groups (Table 4). However, among the categories 
of the cognitive domain, patients in group $\mathrm{S}$ showed significantly higher percentages of recovery in the 'digits backward' category 15 minutes after surgery than in group $\mathrm{N}(67.5 \%$ vs. $43.2 \%, P=0.02)$. With the exception of the latter, no other categories showed significant differences in cognitive domains between the two groups (Table 4).

Table 4 Percentages of recovered patients in each category of the emotional and cognitive domains at 15 and 40 minutes after surgery.

\begin{tabular}{|c|c|c|c|}
\hline & group $\mathrm{N}, n=44$ & group $\mathrm{S}, n=40$ & P-value \\
\hline \multicolumn{4}{|c|}{15 minutes after surgery } \\
\hline \multicolumn{4}{|c|}{ Emotional } \\
\hline Depression & 100 & 97.5 & 0.476 \\
\hline Anxiety & 97.7 & 100 & $>0.999$ \\
\hline \multicolumn{4}{|l|}{ Cognitive } \\
\hline Orientation & 93.2 & 100 & 0.243 \\
\hline Digits forward & 68.2 & 80 & 0.219 \\
\hline Digits backward & 43.2 & 67.5 & $0.025^{*}$ \\
\hline Word generation & 72.7 & 82.5 & 0.282 \\
\hline Recall & 70.5 & 75 & 0.641 \\
\hline \multicolumn{4}{|c|}{40 minutes after surgery } \\
\hline \multicolumn{4}{|c|}{ Emotional } \\
\hline Depression & 100 & 100 & - \\
\hline Anxiety & 100 & 100 & - \\
\hline \multicolumn{4}{|l|}{ Cognitive } \\
\hline Orientation & 95.5 & 97.5 & $>0.999$ \\
\hline Digits forward & 86.4 & 97.5 & 0.112 \\
\hline Digits backward & 84.1 & 97.5 & 0.060 \\
\hline Word generation & 95.5 & 100 & 0.495 \\
\hline Recall & 90.9 & 92.5 & $>0.999$ \\
\hline \multicolumn{4}{|c|}{$\begin{array}{l}\text { Values are percentage of recovered patients. group } \mathrm{N} \text {, neostigmine group; group } \mathrm{S} \text {, } \\
\text { sugammadex group. } * \mathrm{P}<0.05 \text { between groups. }\end{array}$} \\
\hline \multicolumn{4}{|c|}{ Patients' ratings on global perspective on post-operative day 1 are shown in Table 5 . The } \\
\hline \multirow{2}{*}{\multicolumn{4}{|c|}{$\begin{array}{l}\text { patients' global perspective on post-operative day } 1 \text { showed no significant differences in } \\
\text { terms of daily activities, working capacity, and clarity of thought. More patients in group S }\end{array}$}} \\
\hline & & & \\
\hline \multicolumn{4}{|c|}{ were completely satisfied with the anaesthetic care, although this was not statistically } \\
\hline
\end{tabular}


Table 5 Patients' rating on global perspective and satisfaction regarding anaesthetic care on the post-operative day 1 .

\begin{tabular}{|c|c|c|c|}
\hline & group $\mathrm{N}, n=44$ & group $\mathrm{S}, n=40$ & P-value \\
\hline Daily activities & $38(86 \%)$ & $38(95 \%)$ & 0.269 \\
\hline Working capacity & $44(100 \%)$ & $44(100 \%)$ & - \\
\hline Clarity of thought & $44(100 \%)$ & $44(100 \%)$ & - \\
\hline Satisfaction with anaesthetic care & $1 / 19 / 24$ & $0 / 14 / 26$ & 0.438 \\
\hline
\end{tabular}




\section{Discussion}

We performed a prospective, double-blinded, randomized, controlled assessment to demonstrate the influence of reversal with sugammadex or neostgmine on the quality of postoperative recovery using PQRS following a single bolus dose of rocuronium in patients undergoing TPPV with general anaesthesia. In the present study, sugammadex could contribute to favourable initial post-operative recovery in the physiological domains evaluated with the PQRS in patients undergoing day-surgery such as TPPV, as compared with neostigmine.

In a previous retrospective study regarding recovery assessed using PQRS following neostigmine and sugammadex administration, the recovery of the physiological domain at 40 minutes after surgery was significantly higher in group S, which was statistically relevant with the percentage of patients who were fully awake. [7] However, in the present study, even though there was a statistical difference between the 2 groups in the recovery of the physiological domain at 15 minutes after surgery, no single responsible factor was found for this difference in each category of the physiological domain.

Instead, almost every patient in group S had a complete recovery in the categories that consisted of the physiological domain, while there were some patients with no recovery in group N. Thus, these results suggest that sugammadex may be associated with a better postoperative recovery than neostigmine.

Sugammadex has been reported to be superior at preserving haemodynamic stability than anticholinesterases, which have been the most commonly used NM blockade reversal agent to date. $[15,16]$ Due to the fear of adverse effects from cholinesterase inhibitors, these have been associated with concomitant used of anticholinergic agents such as atropine or glycopyrrolate. [17,18] However, the combination of these 2 agents having different pharmacokinetic and pharmacodynamic properties cannot completely prevent their adverse 
effects nor maximize their desired effect. In contrast, as a selective antidote of NM blocking agents, sugammadex may theoretically be better at preserving haemodynamic stability. Conversely, there were no differences in the physiological domain between the two groups 40 minutes after surgery.

These discrepancies of the faster recovery of PQRS between the present study and the previous by Amorim and colleagues might be caused due to the differences in the study design as follows; the degree of NM blockade when reversal agents were given, the doses of administered NM blocking agents, different kinds of NM blocking agents, and various types of surgery such as open or laparoscopy vs sing type-TPPV, which is rarely accompanied by bleeding or systemic complications.

The nociceptive domain in the PQRS includes an assessment of post-operative pain and PONV at different time points. [12] Previous reports have described post-operative pain relief and the use of sugammadex. $[19,20]$ However, according to these reports, differences in pain relief may have resulted from the effects of neostigmine on gastrointestinal motility, which may cause additional pain after gastrointestinal surgery, and to the use of succinylcholine, which may be associated with more myalgia because of fasciculation. In this study, neither gastrointestinal surgery nor succinylcholine use was relevant. Therefore, consistent with the result of other studies on post-operative quality of recovery, there was no difference in pain recovery between the two groups across all time points. [7,21]

An advantage of sugammadex for PONV has also been reported by several studies. $[8,22,23]$ Cholinergic effects from the use of cholinesterase inhibitors can cause a decrease in oesophageal tone, and an increase in secretion and intestinal movement that might be associated with the increased PONV. However, due to the fear of these potential side effects, anticholinergic agents have been used to compensate for these unwanted cholinergic effects. Previous studies have reported that the incidence of PONV was not significantly 
increased after surgery when a cholinesterase inhibitor was used concomitantly with the anticholinergic agents. [24,25] Although certain types of surgery may worsen PONV, TPPV does not appear to be a procedure that aggravates PONV. [26] In the current study, the incidence of PONV was very low in both groups $(2.3 \%$ and $2.5 \%$ in groups $\mathrm{N}$ and $\mathrm{S}$, respectively). This low incidence could be a potential reason for the decreased power of detecting differences in PONV between the 2 groups. Therefore, a clinical trial evaluating PONV from high-risk surgery might provide additional information to clarify the potential protective effects of sugammadex on PONV.

A correlation between sugammadex and a faster recovery of consciousness has been reported. [7,9] However, studies using electroencephalogram-based indices have shown that there were no significant changes after the administration of sugammadex. [11,27] Of the categories of the physiological domain in the PQRS, the recovery of consciousness was also evaluated separately in this study. Our results show there was no significant difference in the recovery of consciousness between the 2 groups across time points. This might be interpreted as sugammadex not having any advantage on improving recovery of consciousness compared with neostigmine. However, the recovery rate of consciousness was very high in both groups as soon as 15 minutes after surgery in this study $(95.5 \%$ and $100 \%$ in groups $\mathrm{N}$ and $\mathrm{S}$, respectively). As we reversed the NM blockade after confirmation of a TOF ratio at 0.5 or greater (shallow block status), the patients in our study may have been more prone to fully recover 15 minutes after surgery. However, the time to extubation was significantly shorter in group $\mathrm{S}$ than in group $\mathrm{N}$ according to the results of this study. Because we only extubated patients who had complete recovery from both consciousness and neuromuscular blockade, sugammadex might be associated with the faster recovery of consciousness in the immediate post-operative period. Further study using different time points of reversal or surgery types should be undertaken to clarify the relationship between the recovery of consciousness and 
use of sugammadex.

The association between the use of sugammadex and the recovery of cognitive function has been demonstrated. [10,28] Previous studies showed no advantage of using sugammadex over cholinesterase inhibitors for NM blockade reversal on cognitive function after surgery. Consistent with previous reports, there were no significant differences in the post-operative recovery of overall cognitive domain in the present study. However, patients in the group $\mathrm{S}$ could recall more numbers in reverse order at 15 minutes after surgery. As can be deduced from the lowest recovery rate, the backward digit span is the most difficult test among the tests used to evaluate the cognitive domain of the PQRS. Thus, this test may be the most sensitive for detecting differences in the recovery of cognitive function. Acetylcholine has been reported to be closely associated with cognitive function [29] and improvements in cognitive function have been reported altering levels of brain acetylcholine levels. [30] Both cholinesterase inhibitors and sugammadex alter acetylcholine levels not only at the peripheral NM junction, but also centrally. Further research focusing on differences in cognitive function may provide additional clarification.

Unfortunately, there are some limitations to the present study. First, there is the short observation period; the assessment of PQRS was not performed after post-operative day 1 . Thus, differences in long-term recovery between the two groups could not be assessed. However, the assessment result after post-operative day 1 would have been similar, as most patients were fully recovered and showed no differences between the groups already on postoperative day 1 . Another limitation is the surgery type. Because the surgery we evaluated was minimally invasive, recovery rates for every PQRS domains were very high in both groups across all time points. Such a high recovery rate in both groups was not very helpful for detection of differences in post-operative recovery between the two groups. So, surgical factors could be a bias for the outcome in more invasive surgery. Finally, because the 
majority of patients were relatively healthy patients with ASA 1-2 in present study, the results may have other outcomes in patients with several underlying disease. Therefore, further study on patients with comorbidities greater than or equal to ASA 3 may be required. 


\section{Conclusions}

In conclusion, reversal with sugammadex following a single bolus dose of rocuronium may be helpful to improve post-operative physiological recovery in the early post-operative period, as assessed by the PQRS in patients undergoing TPPV under general anaesthesia compared with neostigmine. However, further randomised controlled studies with different conditions are necessary. 
Author Contributions: Conceptualization, S.-J. B, J.C.K and N.Y.K ; Data Curation, K.Y.L; Formal analysis, J.H.H; Investigation, K.Y.L , S.-S. K, and H.J.N; Resource, J.H.H; Supervision, J.C.K and N.Y.K; Validation, K.Y.L and S.-S. K; Writing-original draft, S.-J. B ; Writing-review \& editing, J.C.K and N.Y.K All authors read and approved the final manuscript.

Funding: The authors have no financial relationships relevant to this article to disclose.

Acknowledgments: The authors thank Dong-Su Jang, MFA (medical illustrator, Medical Research Support Section, Yonsei University College of Medicine) for his help with the figures.

Conflicts of Interest: The Authors declare that there is no conflict of interest. 


\section{References}

1. Poitras, S.; Beaule, P.E.; Dervin, G.F. Validity of a short-term quality of life questionnaire in patients undergoing joint replacement: The quality of recovery-40. $J$. Arthroplasty 2012, 27, 1604-1608.e1601.

2. Van Herck, P.; Vanhaecht, K.; Deneckere, S.; Bellemans, J.; Panella, M.; Barbieri, A.; Sermeus, W. Key interventions and outcomes in joint arthroplasty clinical pathways: A systematic review. J. Eval Clin. Pract. 2010, 16, 39-49.

3. Cullen, K.A.; Hall, M.J.; Golosinskiy, A. Ambulatory surgery in the united states, 2006. Natl. Health Stat. Report 2009, 1-25.

4. Donati, F. Sugammadex: A cyclodextrin to reverse neuromuscular blockade in anaesthesia. Expert Opin. Pharmacother. 2008, 9, 1375-1386.

5. Loupec, T.; Frasca, D.; Rousseau, N.; Faure, J.P.; Mimoz, O.; Debaene, B. Appropriate dosing of sugammadex to reverse deep rocuronium-induced neuromuscular blockade in morbidly obese patients. Anaesthesia 2016, 71, 265-272.

6. Rex, C.; Bergner, U.A.; Puhringer, F.K. Sugammadex: A selective relaxant-binding agent providing rapid reversal. Curr. Opin. Anaesthesiol. 2010, 23, 461-465.

7. Amorim, P.; Lagarto, F.; Gomes, B.; Esteves, S.; Bismarck, J.; Rodrigues, N.; Nogueira, M. Neostigmine vs. Sugammadex: Observational cohort study comparing the quality of recovery using the postoperative quality recovery scale. Acta Anaesthesiol. Scand. 2014, 58, 1101-1110.

8. Yagan, O.; Tas, N.; Mutlu, T.; Hanci, V. Comparison of the effects of sugammadex and neostigmine on postoperative nausea and vomiting. Braz. J. Anesthesiol. 2017, 67, $147-$ 152.

9. Chazot, T.; Dumont, G.; Le Guen, M.; Hausser-Hauw, C.; Liu, N.; Fischler, M. Sugammadex administration results in arousal from intravenous anaesthesia: A clinical and electroencephalographic observation. Br. J. Anaesth. 2011, 106, 914-916.

10. Batistaki, C.; Riga, M.; Zafeiropoulou, F.; Lyrakos, G.; Kostopanagiotou, G.; Matsota, P. Effect of sugammadex versus neostigmine/atropine combination on postoperative cognitive dysfunction after elective surgery. Anaesth Intensive Care 2017, 45, 581-588.

11. Illman, H.; Antila, H.; Olkkola, K.T. Reversal of neuromuscular blockade by sugammadex does not affect eeg derived indices of depth of anesthesia. J. Clin. Monit. Comput. 2010, 24, 371-376.

12. Royse, C.F.; Newman, S.; Chung, F.; Stygall, J.; McKay, R.E.; Boldt, J.; Servin, F.S.; 
Hurtado, I.; Hannallah, R.; Yu, B. et al. Development and feasibility of a scale to assess postoperative recovery: The post-operative quality recovery scale. Anesthesiology 2010, $113,892-905$.

13. Jildenstal, P.; Eriksson, J.; Warren Stomberg, M.; Jakobsson, J.G. Evaluation of the postoperative quality of recovery scale test and re-test in swedish among healthy volunteers. F1000Res. 2016, 5, 2549.

14. Naito, Y.; Tanaka, Y.; Sasaoka, N.; Iwata, T.; Fujimoto, Y.; Okamoto, N.; Inoue, S.; Kawaguchi, M. Feasibility, reliability, and validity of the japanese version of the postoperative quality of recovery scale: A first pilot study. J. Anesth. 2015, 29, 463-466.

15. Kizilay, D.; Dal, D.; Saracoglu, K.T.; Eti, Z.; Gogus, F.Y. Comparison of neostigmine and sugammadex for hemodynamic parameters in cardiac patients undergoing noncardiac surgery. J. Clin. Anesth. 2016, 28, 30-35.

16. Park, E.S.; Lim, B.G.; Lee, W.J.; Lee, I.O. Sugammadex facilitates early recovery after surgery even in the absence of neuromuscular monitoring in patients undergoing laryngeal microsurgery: A single-center retrospective study. BMC Anesthesiol. 2016, 16, 48.

17. Arsura, E.L.; Brunner, N.G.; Namba, T.; Grob, D. Adverse cardiovascular effects of anticholinesterase medications. Am. J. Med. Sci. 1987, 293, 18-23.

18. Mirakhur, R.K.; Briggs, L.P.; Clarke, R.S.; Dundee, J.W.; Johnston, H.M. Comparison of atropine and glycopyrrolate in a mixture with pyridostigmine for the antagonism of neuromuscular block. Br. J. Anaesth. 1981, 53, 1315-1320.

19. Castro, D.S., Jr.; Leao, P.; Borges, S.; Gomes, L.; Pacheco, M.; Figueiredo, P. Sugammadex reduces postoperative pain after laparoscopic bariatric surgery: A randomized trial. Surg. Laparosc. Endosc. Percutan. Tech. 2014, 24, 420-423.

20. Saricicek, V.; Sahin, L.; Bulbul, F.; Ucar, S.; Sahin, M. Does rocuronium-sugammadex reduce myalgia and headache after electroconvulsive therapy in patients with major depression? J. ECT 2014, 30, 30-34.

21. Baumuller, E.; Schaller, S.J.; Chiquito Lama, Y.; Frick, C.G.; Bauhofer, T.; Eikermann, M.; Fink, H.; Blobner, M. Postoperative impairment of motor function at train-of-four ratio $\geq 0.9$ cannot be improved by sugammadex (1 mg kg-1). British journal of anaesthesia 2015, 114, 785-793.

22. Koyuncu, O.; Turhanoglu, S.; Ozbakis Akkurt, C.; Karcioglu, M.; Ozkan, M.; Ozer, C.; 
Sessler, D.I.; Turan, A. Comparison of sugammadex and conventional reversal on postoperative nausea and vomiting: A randomized, blinded trial. J. Clin. Anesth. 2015, 27, 51-56.

23. Lee, O.H.; Choi, G.J.; Kang, H.; Baek, C.W.; Jung, Y.H.; Woo, Y.C.; Oh, J.; Park, Y.H. Effects of sugammadex vs. Pyridostigmine-glycopyrrolate on post-operative nausea and vomiting: Propensity score matching. Acta Anaesthesiol. Scand. 2017, 61, 39-45.

24. Braun, G.G.; Schywalsky, M.; Wölfel, L.; Müller, H.; Danner, U.; Albert, R. [a comparison of the two anticholinergic agents atropine and glycopyrrolate during antagonism of a muscle relaxation with pyridostigmine]. Anaesthesiologie und Reanimation 1993, 18, 120-122, 125-127.

25. Cheng, C.R.; Sessler, D.I.; Apfel, C.C. Does neostigmine administration produce a clinically important increase in postoperative nausea and vomiting? Anesth. Analg. 2005, $101,1349-1355$.

26. Myklejord, D.J.; Yao, L.; Liang, H.; Glurich, I. Consensus guideline adoption for managing postoperative nausea and vomiting. WMJ 2012, 111, 207-213.

27. Fassoulaki, A.; Chondrogiannis, K.; Staikou, C. Sugammadex at both high and low doses does not affect the depth of anesthesia or hemodynamics: A randomized double blind trial. J. Clin. Monit. Comput. 2017, 31, 297-302.

28. Piskin, O.; Kucukosman, G.; Altun, D.U.; Cimencan, M.; Ozen, B.; Aydin, B.G.; Okyay, R.D.; Ayoglu, H.; Turan, I.O. The effect of sugammadex on postoperative cognitive function and recovery. Braz. J. Anesthesiol. 2016, 66, 376-382.

29. Giovannini, M.G.; Lana, D.; Pepeu, G. The integrated role of ach, erk and mtor in the mechanisms of hippocampal inhibitory avoidance memory. Neurobiol. Learn. Mem. 2015, 119, 18-33.

30. Zaninotto, A.L.; Bueno, O.F.; Pradella-Hallinan, M.; Tufik, S.; Rusted, J.; Stough, C.; Pompeia, S. Acute cognitive effects of donepezil in young, healthy volunteers. Hum. Psychopharmacol. 2009, 24, 453-464. 